\title{
Tuning of Silicon-On-Insulator Ring Resonators with Liquid Crystal Cladding using the Longitudinal Field Component
}

\author{
Wout De Cort, ${ }^{1,2, *}$ Jeroen Beeckman, ${ }^{2}$ Richard James, ${ }^{3}$ F. Anibal Fernández, ${ }^{3}$ \\ Roel Baets ${ }^{1}$ and Kristiaan Neyts ${ }^{2}$ \\ ${ }^{1}$ Photonics Research Group, Department of Information Technology, Ghent University \\ 41, Sint-Pietersnieuwstraat, Gent 9000, Belgium \\ ${ }^{2}$ Liquid Crystals and Photonics group, Department of Electronics and Information Systems, \\ Ghent University \\ 41, Sint-Pietersnieuwstraat, Gent 9000, Belgium \\ ${ }^{3}$ Department of Electronics and Electrical Engineering, University College London \\ Torrington Place, London, WC1E 7JE, UK \\ ${ }^{*}$ Corresponding author: wout.decort@intec.ugent.be
}

\begin{abstract}
We show tuning of the resonance wavelength of silicon-on-insulator microring resonators with liquid crystal cladding. The electrooptic effect of the liquid crystal causes a decrease in effective refractive index for the TE-polarized light in the waveguides. Tuning of the liquid crystal birefringence affects primarily the longitudinal component of the electric field. We achieve a tuning range of $0.6 \mathrm{~nm}$. Through simulation and experiment we perform a thourough study of this phenomenon. (c) 2009 Optical Society of America

OCIS codes: $130.7408,160.3710$.
\end{abstract}

Ring resonators are optical devices designed to show resonance for specific wavelengths. In silicon-on-insulator (SOI), they can be very small, exhibit a high Q-factor and have low losses which make them efficient wavelength-filters [1]. Ring resonators with tunable resonance wavelengths are attractive for reconfigurable optical networks and have led to considerable scientific interest. A method to achieve this is to apply an appropriate cladding layer on the chip. The light in the waveguides 'feels' the refractive index of the cladding with its evanescent tails. Nematic liquid crystal (NLC) is an interesting option. It is optically anisotropic, so its refractive index is directionally dependent. A $\Delta n$ of 0.2 is common for LC materials. As the NLC director orientation can be influenced by an electric field, it 
is possible to achieve electrically tunable optical devices based on NLC. Provided that the physical principles of tuning filters with NLC cladding are well understood, an intelligent design can exploit the very large $\Delta n$ to obtain large tuning ranges. In principle, these ranges can be a lot larger than those achieved with thermal tuning or tuning with carrier injection. Devices guiding TE-polarized light can be made extremely small thanks to the strong confinement of the light, allowing for large free spectral ranges. This strong confinement, however, makes tuning with NLC difficult. The field components have short tails extending in the cladding layer. Therefore the change of the director near the surface of the waveguide (where the reorientation of the director is restricted due to anchoring forces) will determine the resonance shift of the devices. Although TE based filters with NLC-cladding have been reported before [2], their operation is not yet fully analysed quantitatively and there is potential for improvement both in the understanding of the tuning mechanism and the tuning range. Confinement of TM-polarized light is relatively weak, which makes devices (typically 10 times bigger than their TE counterparts) more attractive for tuning [3,4], at the expense of a smaller free spectral range. In this work, we start from a configuration consisting of an SOI ring resonator for TE-polarized light covered with NLC. Top and bottom contacts provide an electric field for reorientation of the LC. In this way we are able to address the longitudinal electric field component of the light and shift the resonance wavelengths of the ring resonators to shorter wavelengths. This is a new method of tuning optical ring resonators which results in a tuning range of approximately $0.6 \mathrm{~nm}$. This is significantly larger than the $0.2 \mathrm{~nm}$ reported before [2]. Through simulations with a fully anisotropic mode solver, we analyse the problem in detail and verify the experimental results.

A layer of NLC is sandwiched between an SOI chip and a glassplate. A schematic crosssection is shown in Fig. 1. The chip consists of a $\mathrm{Si}(\mathrm{n}=3.45)$ substrate, a $2 \mu \mathrm{m}$ insulating layer of $\mathrm{SiO}_{2}(\mathrm{n}=1.49)$ and a thin $(220 \mathrm{~nm})$ layer of $\mathrm{Si}$ in which the ring resonators are defined. We use 5CB, a commercially available NLC, as cladding. We attach a glass plate on top of the chip, using UV curable glue mixed with silica spacers. This glass plate is covered with two additional layers: a conducting layer of transparent ITO (Indium Tin Oxide) and a polyimide alignment layer which gives the LC a preferential direction when rubbed. We can reorient the LC with an electric field if we apply a voltage between the ITO layer and the Si substrate. We determine the alignment of LC on structured SOI surfaces by studying an SOI waveguide and grating covered with 5CB. In the inset of Fig. 2 the structure is shown. An SOI waveguide is flanked by several hundred Si rectangles. The structure is covered with 5CB and sealed with a glass plate with a polyimide alignment layer. The LC on the surface of the glass plate is aligned parallel to the direction of the waveguide. From our experience we know that $5 \mathrm{CB}$ aligns in a planar fashion to $\mathrm{Si}$ or $\mathrm{SiO}_{2}$. Fig. 2 shows the image acquired with a polarization microscope with crossed polarizers. The polarizer is 
aligned parallel to the preferential direction defined by the alignment layer. In the regions that remain black, the LC director causes no change of the polarisation as it retains the same orientation from glass plate to chip surface. The bright parallel lines correspond to the gratings next to the waveguide. The waveguide itself also appears dark. The lines are bright because the LC is twisted to align with the grating sections perpendicular to the waveguide. This is a strong indication of the ability of the director to follow the silicon structure down to nanometer scale (see also [5]). The dark fringes in the bright lines are defects between regions where the director twists clockwise and counterclockwise as both are energetically equivalent. The LC configuration in our device is therefore fairly simple. Throughout the whole device, the director is parallel to the substrate and the glass plate. The direction of orientation is defined by the alignment layer except on the surface of the chip, where it follows the ribs of intersecting surfaces. Under a polarization microscope, the microrings with LC cladding can be distinguished. In Fig. 3, the polarizer and analyzer are at an angle of 45 degrees with the alignment direction of the upper alignment layer. White light becomes colored on transmission through the device, allowing the cell thickness to be estimated as $5 \mu \mathrm{m}$. The image becomes dark when voltage is applied, indicating that the LC director reorients to lie vertically. Light does not undergo a change in polarization and the cell appears dark. The optical characterization is performed by sending TE-polarized light in the waveguides next to the ring resonators through grating couplers [6]. We measure the output power for several values of the applied voltage between $0 \mathrm{~V}$ and $30 \mathrm{~V}$. In Fig. 4. the resonance wavelength for each of the voltages is displayed. The output power in function of the wavelength for voltages of $0 \mathrm{~V}$ and $30 \mathrm{~V}$ is shown in the inset. A blue shift in the resonance wavelength of approximately $0.6 \mathrm{~nm}$ is observed. In our configuration, the wavelength shift is more than twice as large as in previously reported experiments [2] with TE-based devices. In the devices we measured, extinction ratios between $3 \mathrm{~dB}$ and $16 \mathrm{~dB}$ were observed. This low value is partly due to the fact that the structures are designed for air and the NLC can enhance coupling between ring and waveguide and partly due to scattering losses. The slight change in shape of the depicted dips was not seen in all rings.

We use a combination of two simulation tools to study and verify the behavior of the devices described above. It is not trivial to calculate the LC orientation in a certain geometry. In this work, we use a variable order calculation [7] based on the minimization of the Landau-de Gennes free energy functional [8]. This model is implemented in a finite-element scheme. With this tool, it is possible to model the behaviour of LC near small structures like optical waveguides in an accurate way. We use a finite element mode solver to calculate the modes in optical waveguides with NLC top cladding. This solver is based on the solution of the variational form of the curl-curl equations of the electric field [9]. As opposed to most commercial tools, this solver is able to take into account the full anisotropy of the LC. We 
calculate the director orientation in a $2 \mu \mathrm{m}$ cladding layer of $5 \mathrm{CB}$ on an SOI waveguide (see box in Fig. 1) for voltages ranging from $0 \mathrm{~V}$ to $15 \mathrm{~V}$. A spatial distribution of the permittivity tensor results. This is taken into the modal solver, which in turn yields the effective index of the TE ground mode for each value of the voltage. The change in the resonance wavelength of the device is given by $\Delta \lambda / \lambda=\Delta n_{\text {eff }} / n_{\text {eff }}$. A downwards shift in the resonant wavelength with increasing voltage is found, in agreement with experiment (see Fig. 5.). At a critical value of the applied voltage a Freedericksz transition occurs resulting in a distortion of the NLC director field. This threshold effect is observed, as is a saturation effect for higher voltages. This is in good agreement with the experimental results. The blueshift may be understood by examining the individual electric field components. We make a horizontal cut through the mode profiles (the dotted line in Fig. 1). The field profiles are shown in Fig. 6. together with the intensity profile of the mode. As the LC switches from an orientation along the z-axis to a position along the y-axis, the $\mathrm{x}$-component of the electric field will not contribute to a change in $n_{\text {eff }}$. Theoretical calculations confirm this. We can see that a considerable part of the z-component of the electric field is located in the LC cladding. This is due to the high index contrast of the material system and the very small dimensions. At high voltages, the LC director is oriented along the y-axis and presents the ordinary refractive index to the $\mathrm{x}$ - and z-axes leading to a smaller $n_{\text {eff }}$ and resulting in the blue shift of the resonance. The y-component is too small to play a significant role in the tuning. We note that for different values of the device thickness and the anchoring strength the magnitude of the shift and the threshold voltage change, which explains the differences between the simulations and experiments.

We have shown resonance tuning in TE microring resonators exploiting the longitudinal component of the electric field. This leads to a considerably wide tuning range of $0.6 \mathrm{~nm}$. By means of LC- and optical simulations we have gained a good understanding of the tuning mechanism. The insights provided in this work form the base of future research towards widely tunable filters with NLC cladding. These devices can be used in a wide range of applications, from tunable add-drops for reconfigurable networks to (bio-)sensing.

W. De Cort acknowledges the Institute for the Promotion of Innovation through Science and Technology in Flanders (IWTVlaanderen) for a specialization grant. Jeroen Beeckman is Postdoctoral Fellow of the Research Foundation Flanders (FWO-Vlaanderen). The work has been carried out in the framework of the IAP project Photonics@be of the Belgian Science Policy. 


\section{References}

[1] P. Dumon, W. Bogaerts, V. Wiaux, J. Wouters, S. Beckx, J. Van Campenhout, D. Taillaert, B. Luysaert, P. Bienstman, D. Van Thourhout and R. Baets, "Low-Loss SOI Photonic Wires and Ring Resonators Fabricated With Deep UV Lithography", IEEE Photonics Technol. Lett. 16, 1328-1330 (2004)

[2] B. Maune, R. Lawson, C. Gunn, A. Scherer, L. Dalton, "Electrically tunable ring resonators incorporating nematic liquid crystals as cladding layers", Appl. Phys. Lett. 83, 4689-4691 (2003)

[3] V.G. Chigrinov, L. Zhou, A.A. Muravsky, A.W.O. Poon , "Electrically tunable microresonators using photoaligned liquid crystals", US Patent Application Publication, US 2007/0258677 A1, Nov. 8, 2007

[4] A. Di Falco, G. Assanto, "Tunable wavelength-selective add-drop in liquid crystals on a silicon microresonator", Opt. Commun. 279, 210-213 (2007)

[5] H. Desmet, K. Neyts, R. Baets, "Modeling nematic liquid crystals in the neigborhood of edges", J. Appl. Phys 98, 123517 (2005)

[6] D. Taillaert, P. Bienstman and R. Baets, "Compact efficient broadband grating coupler for silicon-on-insulator waveguides", Opt. Lett. 29, 2749-2751 (2004)

[7] R. James, E. Willman, F.A. Fernández and S.E. Day, "Finite-element modeling of liquidcrystal hydrodynamics with a variable degree of order", IEEE T. Electron Dev. 53, 1575-1582 (2006)

[8] P.G. de Gennes and J. Prost, The Physics of Liquid Crystals (Oxford: Oxford University Press, 1995)

[9] J. Beeckman, R. James, F. A. Fernández, W. De Cort, P. J. M. Vanbrabant and K. Neyts, "Calculation of fully anisotropic liquid crystal waveguide modes", accepted to J. Lightwave Technol. 


\section{List of Figures}

1 Schematic view of a device consisting of a SOI substrate with LC cladding, sealed off by a glassplate with conducting layer and alignment layer. . . . . .

2 Under a polarisation microscope the surface appears dark except the regions where the grating is located. This indicates local changes in director orientation. The inset shows a schematic view of the structure. . . . . . . . . . .

3 Under a polarization microscope the ring resonators (radius $4 \mu \mathrm{m}$ ) can be clearly seen. The directions of polarizer $(\mathrm{P})$, analizer $(\mathrm{A})$ and rubbing $(\mathrm{R})$ are indicated on the right. . . . . . . . . . . . . . . . . . .

4 Resonance wavelength shift in function of the applied voltage. The inset shows the output power as a function of the wavelength for $0 \mathrm{~V}$ and $30 \mathrm{~V}$. . . . . . . 10

5 Simulated shift of the resonance wavelength in function of applied voltage. .

6 Relative amplitude of the electric field components along the x-coordinate on a line passing through the center of the waveguide. The inset on the left shows the intensity profile of the waveguide mode. 


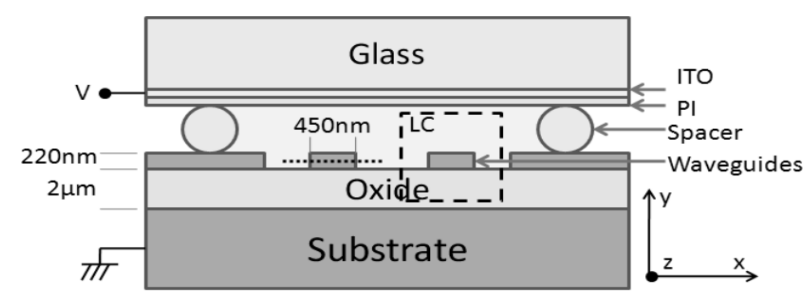

Fig. 1. Schematic view of a device consisting of a SOI substrate with LC cladding, sealed off by a glassplate with conducting layer and alignment layer. 


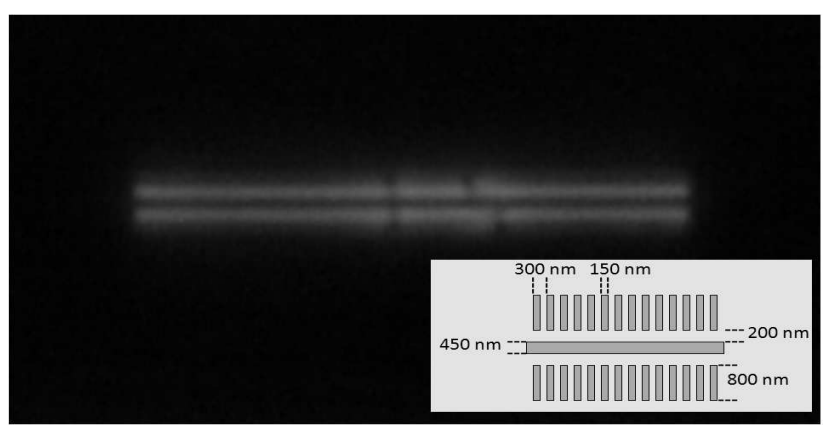

Fig. 2. Under a polarisation microscope the surface appears dark except the regions where the grating is located. This indicates local changes in director orientation. The inset shows a schematic view of the structure. 




Fig. 3. Under a polarization microscope the ring resonators (radius $4 \mu \mathrm{m}$ ) can be clearly seen. The directions of polarizer $(\mathrm{P})$, analizer $(\mathrm{A})$ and rubbing $(\mathrm{R})$ are indicated on the right. 


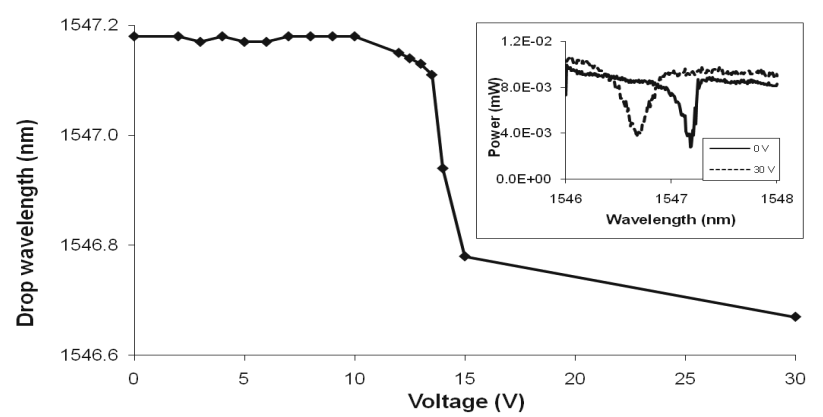

Fig. 4. Resonance wavelength shift in function of the applied voltage. The inset shows the output power as a function of the wavelength for $0 \mathrm{~V}$ and $30 \mathrm{~V}$. 


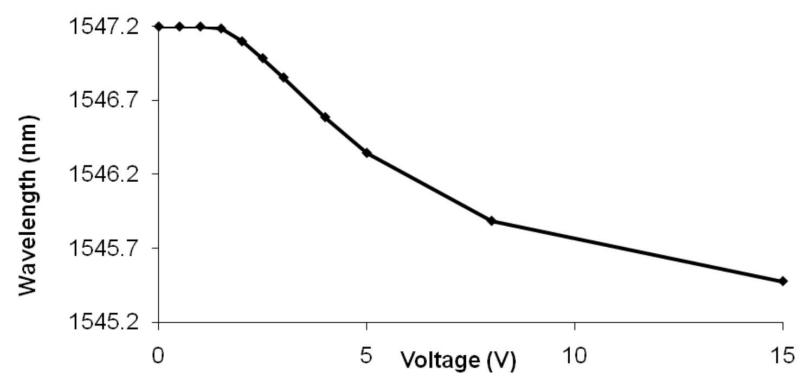

Fig. 5. Simulated shift of the resonance wavelength in function of applied voltage. 


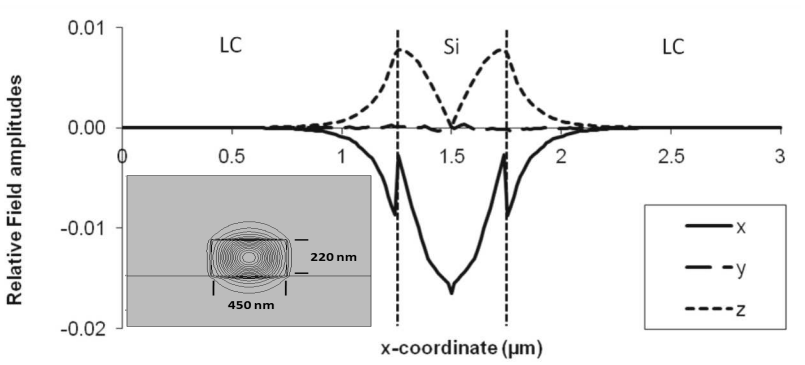

Fig. 6. Relative amplitude of the electric field components along the $\mathrm{x}-$ coordinate on a line passing through the center of the waveguide. The inset on the left shows the intensity profile of the waveguide mode. 